\title{
Micrometeorological Characteristic Study Based on On-line Monitoring System of Transmission Lines
}

\author{
Yuanjing Deng ${ }^{1, a *}$, Yu Zhou ${ }^{2, b}$, Kaiquan Xia ${ }^{1, ~ c}$, Ji Yang ${ }^{1, d}$ \\ ${ }^{1}$ China Electrical Power Research Institute, Beijing, China \\ ${ }^{2}$ Electric Power Research Institute of State Grid Qinghai Electrical Power Company, Xining, China \\ a dengyuanjing@epri.sgcc.com.cn, bzyu1613@qh.sgcc.com.cn, \\ cxiakq@epri.sgcc.com.cn, ${ }^{\mathrm{d}} \mathrm{yangji@epri.sgcc.com.cn}$
}

\begin{abstract}
Keywords: transmission line, smart grid, on-line monitoring, micrometeorological monitoring, Markov Chain-Monte Carlo method

Abstract. The on-line monitoring system undertakes the task to obtain status information of transmission lines and corridors in smart grid. Micrometeorological monitoring is an important resource of environment information of the corridors, which will provide data support for operation and maintenance, forecast, evaluation of transmission lines. Transmission lines in Qinghai depopulated zone is lack of meteorological documents. In order to master micrometeorological characteristics of the transmission line and the corridor environment, processing micrometeorological monitoring data applying Markov Chain Monte Carlo method are carried out in the this paper. Also the suggestion and application of the micrometeorological monitoring data are put forward.
\end{abstract}

\section{Introduction}

Information, automation and interaction are the basic characteristics of smart grid [1], on-line monitoring system of lines and other equipment, ambient environment of power electrical system are important parts of smart grid. The power transmission lines frequently run across high mountains and hills, meteorological environment along the lines is complicate. Meanwhile the nature disaster is one of the main factors to threaten operation safety. Applying the monitoring technology of the transmission lines will provide the real-time monitoring means to the operation and maintenance person to master operation conditions in time, and provide decision support for condition assessment, status warning and disaster forecast of lines.

In order to study the micrometeorological characteristics of transmission lines based on the on-line monitoring system, this paper takes some line as sample, statistic analysis of wind speed, wind direction and air temperature etc meteorological parameters are carried out and suggestion and application of monitoring data are put forward.

\section{Micrometeorological Monitoring of Transmission Lines}

In the area with severe meteorological conditions area, relevant documents are not enough. And there are obvious difference between climate environment and corridor ambient environment. It is necessary of meteorological data in design, operation and maintenance, inspection and re-construction as calculation and decision support.

Most micrometeorological monitoring devices apply modular design, which consist of the power supply, the sensor, the data storage, the communication and the micro-control unit etc. They will collect temperature, humidity, air pressure, wind speed, wind direction and sunshine strength etc environment information, the device have automatic collection and manual collection function. After data are collected, communication methods such as GSM/GPRS/CDMA/3G/4G/WIFI/OPGW can be selected to transmit data to status monitoring agent, which are transmitted to the production management system via the status monitoring access network management after data are summarized. [3] 


\section{Analysis and Study of Micrometeorological Monitoring Data}

Analysis Methods of Micrometeorological Monitoring Data. Statistic analysis of data mainly consists of three steps: selection of distribution type, establishment of distribution parameters, and reasonability analysis of calculation achievement.

Selection of the distribution type consists of two methods, one is value calculation analysis, another is to fit distribution of the monitoring parameters and determine its approximate type and scope according to the observation sample. Because there is certain complication for direct calculation of the monitoring quantity and deducting general possibility distribution function. Therefore second method is generally applied.

Establishment of frequency distribution parameter has two methods: one is matching method, another is Markov Chain Monte Carlo method. Matching method takes some selected frequency distribution type to fit point the monitoring parameters, and make match between the frequency distribution curve and the parameters point ground optimum, quality of fitting can be judged through SPSS software K-S inspection and P-P diagram or Q-Q diagram. Another method is Markov Chain Monte Carlo method (MCMC). It introduces Markov process during random process into Monte Carlo simulation. Basic idea of MCMC is to apply Metropolis sampling algorithm and Gibbs algorithm [5-7] to obtain convergence value of characteristic parameters through establishing random sequence [5].

Take air speed as sample, following types are considered to take for validation distribution of wind speed data: Weibull distribution, positive distribution, exponent Weibull distribution, exponent positive distribution [7]. When Matlab analysis is applied, data in 95\% confidence area are in accordance with Weibull distribution and positive distribution. When it is subtracted its even value and takes the exponent, the obtained data group is in accordance with Weibull distribution under 95\% confidence area and it isn't in accordance with positive distribution. Also the SPSS software can be applied to carry out fitting analysis of its observation and expected possibility.

Apply Weibull distribution $p(v)=\operatorname{Weibull}(k, \lambda)$ and determine its distribution parameter, $\lambda$ and $k$, apply Metropolis sampling algorithm and Gibbs algorithm [6-10].

Concrete steps are: (1) Set initial value $k_{1}^{0}$, Monte Carlo method generates the optional value $k_{0}^{*}=\operatorname{randn}(1) \cdot \sigma$, sample one value at random from positive distribution $\left(0, \sigma^{2}\right)$. (2) Calculate $f(k)=\sum_{t=2}^{343} \frac{k}{\lambda} \cdot\left(\frac{v^{t}}{\lambda}\right)^{k-1} \cdot e^{-\left(\frac{v^{t}}{\lambda}\right)^{k}} \cdot$ (3) Calculate ratio $r=\min \left(e^{\left(f\left(k_{0}^{*}\right)-f\left(k_{1}^{0}\right)\right)}\right) \cdot$ (4) Sample one value $u$ at random for even distribution $(0,1)$. (5) At $u \leq r, k_{1}^{t}=k_{1}^{*}$, otherwise $k_{1}^{t}=k_{1}^{0}$. (6) Carry out iteration, let $\mathrm{t}=\mathrm{t}+1, k_{1}^{0}=a_{1}^{t-1}$, return back $k_{0}^{*}$ and continue circulation. Obtain characteristic parameters $\lambda$ and $k$ after convergence.

For the different Markov chains, select the differential value (i.e., initial value of Markov chain simulation) to carry out several iteration, carry out convergence judgment through simulating whether it is coincided. According to different obeying distribution, inspection method consists of characteristic validation, Chi-square inspection method and Watson inspection method etc of single variable [7][9]. Comparison of fitting results generally takes standard different value to balance.

Statistic analysis of micrometeorological characteristic. Apply Markov chain - Mento Carlo method to carry out metrological parameter model simulation through summarizing all data for 17 sets of micrometeorological monitoring in the line area section, and determine frequency distribution line type and frequency distribution parameters.

For wind direction, its value of distribution angle takes $0-2 \pi$, the winding Cauchy distribution is applied here. Possibility dense function of the winding Cauchy distribution is:

$$
f_{W C}(\theta ; \mu, \gamma)=\frac{1}{2 \pi} \frac{\sinh \gamma}{\cosh \gamma-\cos (\theta-\mu)}(\text { Eq. } 1)
$$


In which, validation distributions of characteristic parameters $u$ and $\lambda$ apply even distribution, i.e., $\mu \sim \operatorname{Unif}(-\pi, \pi), \lambda \sim \operatorname{Unif}(-\pi, \pi)$.

Apply Metropolis sampling algorithm and Gibbs algorithm, obtain parameters $\mu$ and $\lambda$ of the winding Cauchy distribution through calculation after 15000 times sampling circulation, put in into the possibility dense function, and obtain the possibility dense function of wind direction:

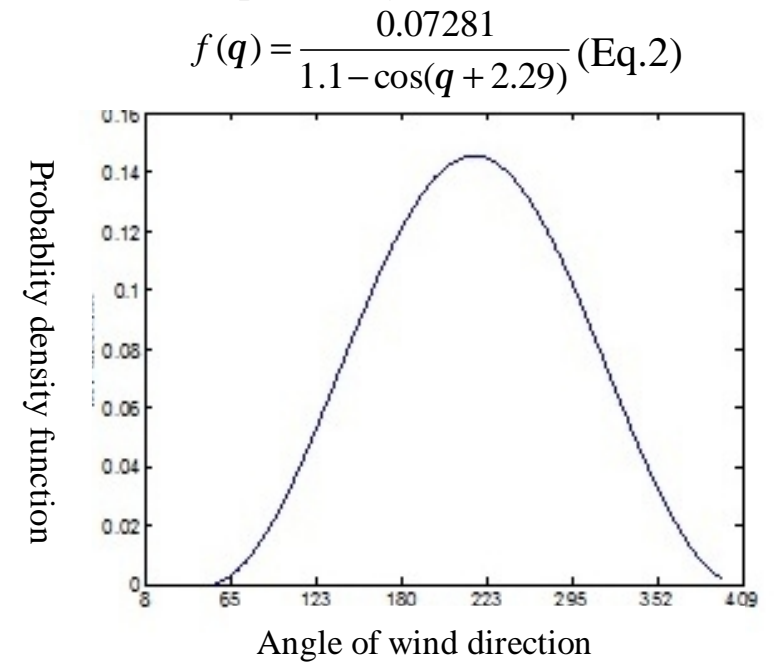

Fig. 1 Wind direction possibility density function of micrometeorological monitoring

For wind speed, in case there is no low wind speed, i.e., $0 \mathrm{~m} / \mathrm{s}$, Weibull distribution is generally applied. Through monitoring of data observed within one year, it doesn't exist when wind speed data approach 0 .

Wind speed distribution is: $p(v)=\operatorname{Weibull}(k, \lambda) \quad$ (Eq.3)

In which, validation distributions of $\mathrm{k}$ and $\lambda$ apply Gammar distribution and positive distribution respectively, i.e.,

$$
k \sim \operatorname{Gammar}\left(1,10^{-3}\right) \lambda \sim \operatorname{Norm}\left(0,10^{3}\right)
$$

Apply Markov chain - Mento Carlo method and obtain wind speed distribution

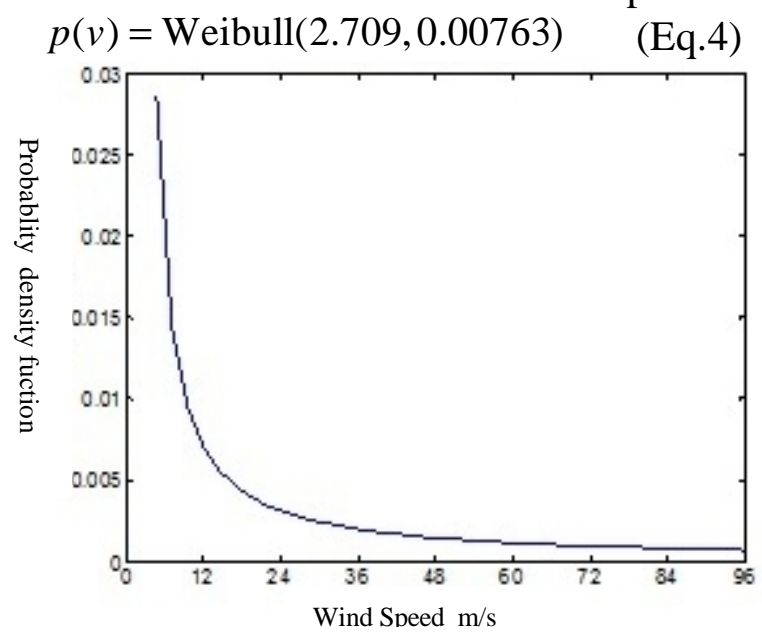

Fig. 2 Wind speed possibility density function of micrometeorological monitoring

For environment temperature, apply positive distribution model for it, i.e., $p(y)=\operatorname{Norm}\left(\mu, \sigma^{2}\right)$. For $\mu$, apply AR model $\mu^{t}=\alpha_{1} y^{t-1}+\alpha_{0}$, validation distribution of $\alpha_{1}, \alpha_{0}, \sigma$ apply positive distribution:

$$
\alpha_{1} \sim \operatorname{Norm}\left(0,10^{4}\right), \alpha_{0} \sim \operatorname{Norm}\left(0,10^{4}\right), \sigma \sim \operatorname{Norm}\left(0,10^{4}\right)
$$
is:

Apply Markov chain - Mento Carlo method for this model, obtain its positive distribution model

$$
Y \sim \operatorname{Norm}\left(0.9578 y_{i-1}+0.1029,6.2150\right)(\text { Eq.5) }
$$




\section{Application of micrometeorological monitoring data}

According to statistic results from 1981 to 2010, nature disaster and equipment fault are two great factors to endanger stability of the grid system. On-line monitoring is first defend line to ensure safe and reliable operation of the transmission lines, meteorological information of the corridors can be obtained in time when the advanced status monitoring technical means are applied, which provides means for real-time monitoring and control of the power transmission line.

The on-line monitoring system can carry out environment auxiliary monitoring and control of the channel in inconvenient traffic or special road section. Data are transmitted back to the status evaluation centre of the equipment by the wireless public network system or OPGW through the micrometeorological monitoring device, the video image monitoring device and the on-line monitoring system, the on-line monitoring system arranged in the status evaluation centre of the equipment can take advantage of the model and operation result at site to judge conditions of the channel in the power transmission line, and alarm in time.

Through long term accumulation of micrometeorological data by the status monitoring system, it will be helpful to carry out analysis and summarization of meteorological environment along the line, provide data support for meteorological load during re-construction of the line, carry out warning study about meteorological disaster by the big data, the nerve network, the expert system etc intelligent analysis technology, identify the relevant meteorological fault characteristic information, improve fault defense capability of the line and promote intelligent level of operation and maintenance management.

\section{Conclusions}

Through statistic analysis of all data of micrometeorological monitoring of transmission lines, obtain local micrometeorological parameters and distribution characteristic of the corridor, obtain the distribution parameters. Temperature difference is very significant, average value of wind speed is very great, and direction takes west wind as prevail.

The methods and steps for analysis of micrometeorological parameters are summarized, and take micrometeorological monitoring data of the concrete line as sample, and carry out distribution calculation of wind direction, wind speed and air temperature. In which, estimation of the distribution parameters applies Markov Chain-Monte Carlo method to obtain characteristic parameters of distribution through circulation iteration by Metropolis sampling algorithm and Gibbs algorithm.

Because area covering by the transmission lines is brand, geological environment is complicated, it is suggested to promote quality of on-line monitoring device on the transmission lines so as to suit severe environment, strengthen and optimize micrometeorological on-line monitoring, accumulate micrometeorological data along the line, and provide data foundation for re-construction, operation and maintenance, disaster warning of the lines in future.

\section{Acknowledgements}

This work was financially supported by Project of State Grid Corporation of China (Study on Reliability Technology of Smart On-line Monitoring on Transmission Lines).

\section{References}

[1] Liu Zhenya etc. Smart Grid Technology[M]. Beijing: China Electric Power Press. 2010.

[2] Luo Shengzhou, Wang Qingchun, Dai Sheng. Analysis of several climate characteristics of meteorological disasters in Qinghai province[J]. Glaciology and Geocryology, 2012,34(6):1380-1386.

[3] Huang Xinbo etc. On-line monitoring and fault diagnosis of power transmission line[M]. Beijing: China Electric Power Press, 2014. 
[4] Production and technology department of State Grid. Technical standard summary of equipment in grid[M]. Beijing: China Electric Power Press, 2012.

[5] Shi Huifeng. Load forecast model of MCMC optimization parameter based on Bayes theory[D]. Beijing: North China Electric Power University, 2013.

[7] Luo Gang,Shi Dongyuan,Chen Jinfu etc, MCMC method for time sequence simulation of wind and light power generation [J]. Grid technology, 2014,38(2):321-327

[8] Gelman A,Carlin J, Stern H,et al.Baysesian data analysis[M].London,UK:Chapman\& Hall,1995.

[9] Wang Kongsen Sheng Yigao Wang Kui etc. Operation risk evaluation about dynamic capacity enhancement of power transmission line [J]. Power system automation, 2011,35(23):11-15.

[10]Lenk P. Bayesian inference and Markov chain Monte Carlo[M].USA University of Michigan,2001. 